\title{
FACTORIZATION PROBLEM WITH INTERSECTION
}

\author{
R.A. ATNAGULOVA, O.V. SOKOLOVA
}

\begin{abstract}
We propose a generalization of the factorization method to the case when $\mathcal{G}$ is a finite-dimensional Lie algebra $\mathcal{G}=\mathcal{G}_{0} \oplus M \oplus N$ (direct sum of vector spaces), where $\mathcal{G}_{0}$ is a subalgebra in $\mathcal{G}, M, N$ are $\mathcal{G}_{0}$-modules, and $\mathcal{G}_{0}+M, \mathcal{G}_{0}+N$ are subalgebras in $\mathcal{G}$. In particular, our construction involves the case when $\mathcal{G}$ is a $\mathbb{Z}$-graded Lie algebra. Using this generalization, we construct certain top-like systems related to algebra $s o(3,1)$. According to the general scheme, these systems can be reduced to solving systems of linear equations with variable coefficients. For these systems we find polynomial first integrals and infinitesimal symmetries.
\end{abstract}

Keywords: factorization method, Lie algebra, integrable dynamical systems.

Mathematics Subject Classification: 17B80

\section{INTRODUCTION}

The classical factorization method [1, 2, 3, 4] (called also Adler-Kostant-Symes scheme) allows us to integrate the following special system of ordinary differential equations

$$
U_{t}=\left[U_{+}, U\right], \quad U(0)=U_{0} .
$$

Here $U(t)$ is a function with values in Lie algebra $\mathcal{G}$ being a direct sum of vector spaces $\mathcal{G}_{+}$and $\mathcal{G}_{-}$. Each of these spaces is a subalgebra in $\mathcal{G}$. By $U_{+}$we denote the projection of $U$ on $\mathcal{G}_{+}$. For the sake of simplicity we assume that $\mathcal{G}$ is embedded into the matrix algebra.

The solution to problem (1) is given by the formula

$$
U(t)=A(t) U_{0} A^{-1}(t) .
$$

Here matrix $A(t)$ is defined as a solution to the factorization problem

$$
A^{-1} B=\exp \left(-U_{0} t\right), \quad A \in G_{+}, \quad B \in G_{-},
$$

where $G_{+}$and $G_{-}$are the Lie groups of algebras $\mathcal{G}_{+}$and $\mathcal{G}_{-}$. If $\mathcal{G}_{-}$is an ideal, the factorization problem can be solved explicitly $A=\exp \left(\left(U_{0}\right)_{+} t\right), B=A \exp \left(-U_{0} t\right)$. In the case $G_{+}$and $G_{-}$ are algebraic groups, the conditions $A \in G_{+}$and $A \exp \left(-U_{0} t\right) \in G_{-}$are a system of algebraic equations determining matrix $A$ uniquely for $t$ close to zero.

It was shown in work [4] that the classical factorization problem can be reduced to the system of linear ordinary differential equations with variable coefficients. Moreover, in work [4] the factorization method can be generalized for the case

$$
\mathcal{G}=V_{1} \oplus V_{2},
$$

where $V_{1}, V_{2}$ are some vector subspaces belonging respectively to subalgebras $\mathcal{G}_{+}$and $\mathcal{G}_{-}$. It was shown that if

$$
\left[\mathcal{G}_{+} \cap \mathcal{G}_{-}, V_{i}\right] \subset V_{i}, \quad i=1,2,
$$

R.A. Atnagulova, O.V. Sokolova, Factorization Problem with Intersection.

(C) Atnagulova R.A., Sokolova O.V. 2014.

Submitted September 2, 2013. 
then integrating equation (1), where "+" indicates the projection on $V_{1}$ parallel to $V_{2}$, can be also reduced to solving a system of linear ordinary differential equations with variable coefficients.

Let us recall the construction of Golubchik-Sokolov. In [4] the authors considered a factorization problem with the right logarithmic derivatives of $A$ and $B$ involved:

$$
A^{-1} B=Z(t), \quad A_{t} A^{-1} \in V_{1}, \quad B_{t} B^{-1} \in V_{2}, \quad Z(0)=A(0)=B(0)=E,
$$

and $V_{i}$ are arbitrary vector space satisfying (4). Let $U(t)=A(t) q(t) A^{-1}(t)$, where $q(t)=$ $-Z_{t} Z^{-1}$. One can see that this function satisfies the equation

$$
U_{t}=\left[U_{+}, U\right]+A q_{t} A^{-1} .
$$

We observe that if one lets $Z(t)=\exp \left(-U_{0} t\right)$, then $q(t)=U_{0}, q_{t}=0$, and equation (7) coincides with (1). Thus, solving of the factorization problem is related with solving a nonlinear differential equation (1).

Then the following factorization problem

$$
\alpha^{-1} \beta=Z(t), \quad \alpha^{-1} \alpha_{t} \in V_{1}, \quad \beta^{-1} \beta_{t} \in V_{2}, \quad Z(0)=\alpha(0)=\beta(0)=E,
$$

was considered, where both logarithmic derivatives are left. This problem is not connected directly with equations like (1), but it can be reduced to a linear equation with variable coefficients. Namely, in work [4] there was considered a linear mapping $L(t): V_{1} \rightarrow V_{1}$ defined by the formula

$$
L(t)(v)=\left(Z^{-1}(t) v Z(t)\right)_{+}
$$

Since $L(0)$ is the identity mapping, $L(t)$ is invertible for small $t$. Then it was shown in this work that a solution $\alpha$ to linear equation

$$
\alpha_{t}=-\alpha L^{-1}(t)\left(\left(Z^{-1} Z_{t}\right)_{+}\right), \quad \alpha(0)=E,
$$

and function $\beta$ defined as

$$
\beta=\alpha Z(t)
$$

are the unique solution to factorization problem (8).

The final step of construction is that under additional assumption (5) there was established a link between two factorization problems. Namely, let $V_{1} \subset \mathcal{G}_{+}, V_{2} \subset \mathcal{G}_{-}$, where $\mathcal{G}_{+}$and $\mathcal{G}_{-}$ are Lie subalgebras of algebra $\mathcal{G}$ such that $\mathcal{G}_{+} \cap \mathcal{G}_{-}=\mathcal{G}_{0} \neq\{0\}$. Then solutions to problems (6) and (8) satisfy the same factorization problem

$$
A^{-1} B=Z(t), \quad A \in G_{+}, \quad B \in G_{-}, \quad A(0)=B(0)=E,
$$

where $G_{+}$and $G_{-}$are the Lie groups of algebras $\mathcal{G}_{+}$and $\mathcal{G}_{-}$. Since $\mathcal{G}_{0} \neq\{0\}$, problem (11) is not uniquely solvable. Let $\alpha, \beta$ be the solution to factorization problem (8). Since it also solves problem (11), all other solutions to problem (11) can be obtained by the relation

$$
A=H \alpha, \quad B=H \beta, \quad H(0)=E,
$$

where $H$ is an arbitrary element in Lie group $G_{0}$ of Lie algebra $\mathcal{G}_{0}$.

It was shown in work [4] that in order to $A, B$ satisfy factorization problem (6), $H$ should solve the following linear equation

$$
H_{t}=-H\left(\left(\alpha_{t} \alpha^{-1}\right)_{-}+\left(\beta_{t} \beta^{-1}\right)_{+}\right), \quad H(0)=E .
$$

Thus, having solved equation (9) for $\alpha$, we can find function $\beta$ by relation (10). Then, having solved equation (13), by (12) we can find solution $A, B$ to factorization problem (6). In the last step we can write out the solution to equation (7) employing formula (2).

In the most part of works devoted to the factorization problem there was treated the case when algebra $\mathcal{G}$ can be expanded into the sum of two subspaces. In particular, in work [4] I.Z. Golubchik and V.V. Sokolov constructed a scheme for such case. The aim of the present work is to generalized their construction for the case, when algebra $\mathcal{G}$ is a sum of three subspaces. 
This generalization allows one to solve a wider class of integrable systems of ODEs by the factorization problem method.

In Section 2 we construct the generalization of the factorization method for the case when $\mathcal{G}$ is a finite-dimensional Lie algebra, $\mathcal{G}=\mathcal{G}_{0} \oplus M \oplus N$ is a direct sum of vector spaces, where $\mathcal{G}_{0}$ is a subalgebra in $\mathcal{G}$, and $M, N$ are $\mathcal{G}_{0}$-modules, $\mathcal{G}_{0}+M, \mathcal{G}_{0}+N$ are subalgebras in $\mathcal{G}$. This construction comprises an important particular case when $\mathcal{G}$ is a graded Lie algebra.

In Section 3 we construct some dynamical top-like systems related with Lie algebras $s l(2)$ and $s o(3,1)$. In accordance with general scheme, these systems can be reduced to linear system of ODEs with variable coefficients. For all these systems we find polynomial first integrals and infinitesimal symmetries. It is shown that these systems can be integrated by quadrature by means of Lie algorithm. However, they do not satisfy Painlevé test since they possess movable branching points.

\section{MAIN CONSTRUCTION}

Assume we are given the expansion

$$
\mathcal{G}=\mathcal{G}_{0} \oplus M \oplus N
$$

of a finite-dimensional Lie algebra $\mathcal{G}$ over $\mathbb{R}$ into the direct sum (as vector subspaces) of Lie subalgebras $\mathcal{G}_{0}$ and two vector subspaces $M$ and $N$ such that

- $M, N$ are $\mathcal{G}_{0}$-modules;

- $\mathcal{G}_{0}+M, \mathcal{G}_{0}+N$ are subalgebras in $\mathcal{G}$.

Theorem. Let a linear operator $R$ be given by the formula

$$
R(q)=\alpha_{-1} q^{-1}+\alpha_{0} q^{0}+\alpha_{1} q^{1},
$$

where $q=q^{-1}+q^{0}+q^{1}, q^{-1} \in N, q^{0} \in \mathcal{G}_{0}, q^{1} \in M, \alpha_{-1}, \alpha_{0}, \alpha_{1} \in \mathbb{R}$. Then the equation

$$
q_{t}=[R(q), q],\left.\quad q\right|_{t=0}=q_{0},
$$

is reduced to a system of linear differential equations with variable coefficients by GolubchikSokolov construction (cf. Introduction).

Proof. We employ the construction from paper [4]. In order to do it, as $\overline{\mathcal{G}}$ we take a direct sum of three copies of algebra $\mathcal{G}$, i.e.,

$$
\overline{\mathcal{G}}=\mathcal{G} \oplus \mathcal{G} \oplus \mathcal{G} .
$$

As $\overline{\mathcal{G}}_{+}$we take a diagonal subalgebra of algebra $\overline{\mathcal{G}}$ :

$$
\overline{\mathcal{G}}_{+}=\{(a, a, a) \mid a \in \mathcal{G}\},
$$

and as $\overline{\mathcal{G}}_{-}$we take the following subalgebra

$$
\overline{\mathcal{G}}_{-}=\left\{(a, b, c) \mid a \in \mathcal{G}_{0}+N, b \in \mathcal{G}_{0}+M, c \in \mathcal{G}\right\} .
$$

Consider the vector subspace

$$
\bar{M}=\left\{(a, b, c) \mid a \in \mathcal{G}_{0}+N, b \in \mathcal{G}_{0}+M, c \in N+M\right\} .
$$

Then for $\overline{\mathcal{G}}$ and $\overline{\mathcal{G}}_{+}, \bar{M}$ condition (4) is satisfied, i.e., $\overline{\mathcal{G}}=\overline{\mathcal{G}}_{+} \oplus \bar{M}$. Condition (5) is obviously satisfied for subalgebra $\overline{\mathcal{G}}_{+}$. And for $\bar{M}$ it is easy to check that

$$
\left[\left(\overline{\mathcal{G}}_{+} \cap \overline{\mathcal{G}}_{-}\right), \bar{M}\right] \subset \bar{M} \text {. }
$$

Thus, it follows from work [4 that equation

$$
\bar{q}_{t}=\left[\bar{q}_{+}, \bar{q}\right],\left.\quad \bar{q}\right|_{t=0}=\bar{q}_{0},
$$

is reduced to solving a system of linear differential equations with variable coefficients. Here $\bar{q} \in \overline{\mathcal{G}}, \bar{q}_{+}$is the projection of $\bar{q}$ on $\overline{\mathcal{G}}_{+}$parallel to $\bar{M}$. 
To complete the proof, it remains to take $\bar{q}=\left(\alpha_{1} q, \alpha_{-1} q, \alpha_{0} q\right)$, where $q \in \mathcal{G}$. We note that in the present case $\bar{q}_{+}=(R(q), R(q), R(q))$, where operator $R$ is given by formula (14). Now it is easy to see that equation (16) for each component reads as $q_{t}=[R(q), q]$ which coincides with equation (15).

Corollary 1. Let $\mathcal{G}=\bigoplus_{i=-k}^{k} \mathcal{G}_{i}$ be a $\mathbb{Z}$-graded Lie algebra and $N=\bigoplus_{i=-k}^{-1} \mathcal{G}_{i}, M=\bigoplus_{i=1}^{k} \mathcal{G}_{i}$. Then the hypothesis of Theorem holds true and the equation

$$
q_{t}=\left[\alpha_{-1} \sum_{i=-k}^{-1} q_{i}+\alpha_{0} q_{0}+\alpha_{1} \sum_{i=1}^{k} q_{i}, q\right]
$$

is reduced to a system of linear differential equations with variable coefficients.

Remark 1. It follows from formula (15) that the traces $q^{i}, i=1,2, \ldots$, are polynomial first integrals for dynamical system (15). Since $q$ is independent of the spectral parameter, the amount of integrals is not enough for the complete integrability of (15).

Remark 2. For an arbitrary element $g \in \mathcal{G}$, such that $\left[g, \mathcal{G}_{0}\right] \subseteq \mathcal{G}_{0},[g, M] \subseteq M,[g, N] \subseteq N$, we have $[g, R(q)]=R([g, q])$. Thus, $q_{\tau}=[g, q]$ is a linear symmetry for equation (15).

\section{EXAMPLES}

We provide to important examples implied by Corollary 1.

Example 1. On $\mathcal{G}=\mathfrak{s l}_{2}$ we consider the following grading

$$
\mathcal{G}_{-1}=\left\{\left(\begin{array}{ll}
0 & 0 \\
v & 0
\end{array}\right)\right\}, \quad \mathcal{G}_{0}=\left\{\left(\begin{array}{cc}
w & 0 \\
0 & -w
\end{array}\right)\right\}, \quad \mathcal{G}_{1}=\left\{\left(\begin{array}{ll}
0 & u \\
0 & 0
\end{array}\right)\right\} .
$$

Then equation (17) can be rewritten as the system

$$
\left\{\begin{array}{l}
w_{t}=\left(\alpha_{1}-\alpha_{-1}\right) u v \\
u_{t}=2\left(\alpha_{0}-\alpha_{1}\right) u w \\
v_{t}=2\left(\alpha_{-1}-\alpha_{0}\right) v w .
\end{array}\right.
$$

This system possesses the linear infinitesimal symmetry

$$
\left\{\begin{array}{l}
w_{\tau}=0 \\
u_{\tau}=u \\
v_{\tau}=-v
\end{array}\right.
$$

as well as the expected polynomial first integral (cf. Remark 1)

$$
H_{1}=\frac{1}{2} \operatorname{Tr}\left(q^{2}\right)=u v+w^{2} .
$$

We notice that the infinitesimal symmetry is generated by the transformations $u \rightarrow k u, v \rightarrow$ $k^{-1} v, w \rightarrow w$.

System (18) can be easily integrated by various means. Let us demonstrate the Lie algorithm for this simple example. In order to apply this algorithm for a system of ODEs with $n$ variables, it is necessary to have in total $n$ symmetries, (including the original system) and first integrals such that 1) all the symmetries commute; 2) all the first integrals are first integrals for each symmetry. In our case we have two symmetries and one integral $H_{1}$. It is easy to check that $\frac{d H_{1}}{d \tau}=0$. Employing the identity $H_{1}=C$, we exclude $w$ and obtain

$$
\left\{\begin{array} { l } 
{ u _ { t } = 2 ( \alpha _ { 0 } - \alpha _ { 1 } ) u \sqrt { C - u v } } \\
{ v _ { t } = 2 ( \alpha _ { - 1 } - \alpha _ { 0 } ) v \sqrt { C - u v } }
\end{array} \quad \left\{\begin{array}{l}
u_{\tau}=u \\
v_{\tau}=-v
\end{array}\right.\right.
$$


Then we seek for the change of variables $\bar{u}=\varphi(u, v), \bar{v}=\psi(u, v)$ such that

$$
\left\{\begin{array} { l } 
{ \varphi _ { t } = 1 , } \\
{ \psi _ { t } = 0 . }
\end{array} \quad \left\{\begin{array}{l}
\varphi_{\tau}=0 \\
\psi_{\tau}=1 .
\end{array}\right.\right.
$$

It is easy to note that functions $\varphi, \psi$ satisfy the following overdetermined systems of partial differential equations

$$
\begin{aligned}
& \left\{\begin{array}{l}
2\left(\left(\alpha_{0}-\alpha_{1}\right) \varphi_{u} u+\left(\alpha_{-1}-\alpha_{0}\right) \varphi_{v} v\right) \sqrt{C-u v}=1, \\
\varphi_{u} u-\varphi_{v} v=0
\end{array}\right. \\
& \left\{\begin{array}{l}
2\left(\left(\alpha_{0}-\alpha_{1}\right) \psi_{u} u+\left(\alpha_{-1}-\alpha_{0}\right) \psi_{v} v\right) \sqrt{C-u v}=0, \\
\psi_{u} u-\psi_{v} v=1
\end{array}\right.
\end{aligned}
$$

By these identities we find all partial derivatives of $\varphi$ and $\psi$. The existence of functions $\varphi$ and $\psi$ follows from the compatibility of systems (19). Integrating, we obtain the following answer

$$
\begin{gathered}
\varphi(u, v)=\frac{\operatorname{arth} \sqrt{1-\frac{u v}{C}}}{\left(\alpha_{1}-\alpha_{-1}\right) \sqrt{C}}+k_{1}, \\
\psi(u, v)=\frac{\alpha_{0}-\alpha_{-1}}{\alpha_{1}-\alpha_{-1}} \ln \left(\alpha_{1}-\alpha_{-1}\right) u+\frac{\alpha_{0}-\alpha_{1}}{\alpha_{1}-\alpha_{-1}} \ln \left(\alpha_{1}-\alpha_{-1}\right) v+k_{2} .
\end{gathered}
$$

Functions $u(t)$ and $v(t)$ are found by condition $\psi(u, v)=0, \varphi(u, v)=t$. We obtain the solution to the original system

$$
\begin{gathered}
u=z k_{3}\left(\cosh z\left(\alpha_{1}-\alpha_{-1}\right)\left(t-k_{1}\right)\right)^{\frac{2\left(\alpha_{0}-\alpha_{1}\right)}{\alpha_{1}-\alpha_{-1}}}, \\
v=\frac{z}{k_{3}}\left(\cosh z\left(\alpha_{1}-\alpha_{-1}\right)\left(t-k_{1}\right)\right)^{\frac{2\left(\alpha_{0}-\alpha_{-1}\right)}{\alpha_{-1}-\alpha_{1}}}, \\
w=z \tanh z\left(\alpha_{1}-\alpha_{-1}\right)\left(t-k_{1}\right),
\end{gathered}
$$

where $z, k_{1}, k_{3}$ are arbitrary constants. At that, $z^{2}=C$, and $k_{3}$ is related with $k_{1}, k_{2}, C$ by a rather bulky formula which we do not provide. In view of formulae for solution, for the same values of constants $\alpha_{i}$ functions $u(t)$ and $v(t)$ have movable branching points in the complex plane.

In what follows we provide not so trivial examples related with Lie algebra $s o(3,1)$. Integrable Hamiltonian dynamical systems with a quadratic right hand side related with semi-simple Lie algebras were considered in works [5, 6, 7]. From the point of view of applications, one of the most interesting algebras is $s o(4)$ or the complex-isomorphic algebra $s o(3,1)$. Integrable Hamiltonian systems related with these algebras were studied in [8, 9, 10. We provide two new examples related with algebra $s o(3,1)$. The systems in these examples are likely nonHamiltonian. We find a sufficient amount of first integrals and infinitesimal symmetries to claim that they are locally integrable by means of Lie algorithm (cf. Example 1).

Example 2. We take $\mathcal{G}=\left\{A \in \mathbb{R}_{4 \times 4} \mid A^{*}=-A\right\}$, where the involution is defined by the formula $A^{*}=T A^{t} T^{-1}, T=e_{11}+e_{22}+e_{34}+e_{43}$, the superscript $t$ indicates the transposition, and $e_{i j}$ are matrix unities. If we choose $T=e_{11}+e_{22}+e_{33}-e_{44}$, the condition of skew-symmetry with respect to the involution determines algebra $s o(3,1)$. The isomorphism between $\mathcal{G}$ and $s o(3,1)$ is defined by the formula $\bar{q}=B^{-1} q B$, where $q \in \mathcal{G}, \bar{q} \in s o(3,1)$, and matrix $B$ is equal to

$$
e_{11}+e_{22}+\frac{\sqrt{2}}{2}\left(e_{33}+e_{34}+e_{43}-e_{44}\right)
$$


On algebra $\mathcal{G}$ we can introduce 3 -grading. Indeed, a general element of the algebra reads as

$$
q=\left(\begin{array}{cccc}
0 & w_{1} & u_{1} & v_{1} \\
-w_{1} & 0 & u_{2} & v_{2} \\
-v_{1} & -v_{2} & w_{2} & 0 \\
-u_{1} & -u_{2} & 0 & -w_{2}
\end{array}\right)
$$

It is easy to check that the expansion of algebra $\mathcal{G}$ into the sum of components

$$
\begin{aligned}
& \mathcal{G}_{0}=\left\{w_{1}\left(e_{12}-e_{21}\right)+w_{2}\left(e_{33}-e_{44}\right)\right\}, \\
& \mathcal{G}_{1}=\left\{u_{1}\left(e_{13}-e_{41}\right)+u_{2}\left(e_{23}-e_{42}\right)\right\}, \\
& \mathcal{G}_{-1}=\left\{v_{1}\left(e_{14}-e_{31}\right)+v_{2}\left(e_{24}-e_{32}\right)\right\}
\end{aligned}
$$

equips $\mathcal{G}$ by the structure of a 3 -graded Lie algebra.

In this case equation (17) can be rewritten as the system

$$
\left\{\begin{array}{l}
w_{1 t}=\left(\alpha_{1}-\alpha_{-1}\right)\left(u_{2} v_{1}-u_{1} v_{2}\right) \\
u_{1 t}=\left(\alpha_{0}-\alpha_{1}\right)\left(u_{2} w_{1}-u_{1} w_{2}\right) \\
v_{1 t}=\left(\alpha_{0}-\alpha_{-1}\right)\left(v_{2} w_{1}+v_{1} w_{2}\right) \\
w_{2 t}=\left(\alpha_{1}-\alpha_{-1}\right)\left(u_{1} v_{1}+u_{2} v_{2}\right) \\
u_{2 t}=\left(\alpha_{1}-\alpha_{0}\right)\left(u_{1} w_{1}+u_{2} w_{2}\right) \\
v_{2 t}=\left(\alpha_{-1}-\alpha_{0}\right)\left(v_{1} w_{1}-v_{2} w_{2}\right)
\end{array}\right.
$$

This system possesses the following linear and quadratic infinitesimal symmetries

$$
\left\{\begin{array} { l } 
{ w _ { 1 \tau _ { 1 } } = 0 , } \\
{ u _ { 1 \tau _ { 1 } } = - u _ { 1 } , } \\
{ v _ { 1 \tau _ { 1 } } = v _ { 1 } , } \\
{ w _ { 2 \tau _ { 1 } } = 0 , } \\
{ u _ { 2 \tau _ { 1 } } = - u _ { 2 } , } \\
{ v _ { 2 \tau _ { 1 } } = v _ { 2 } . }
\end{array} \quad \left\{\begin{array} { l } 
{ w _ { 1 \tau _ { 2 } } = 0 , } \\
{ u _ { 1 \tau _ { 2 } } = u _ { 2 } , } \\
{ v _ { 1 \tau _ { 2 } } = v _ { 2 } , } \\
{ w _ { 2 \tau _ { 2 } } = 0 , } \\
{ u _ { 2 \tau _ { 2 } } = - u _ { 1 } , } \\
{ v _ { 2 \tau _ { 2 } } = - v _ { 1 } . }
\end{array} \quad \left\{\begin{array}{l}
w_{1 \tau_{3}}=\left(-\alpha_{1}+\alpha_{-1}\right)\left(u_{1} v_{1}+u_{2} v_{2}\right), \\
u_{1 \tau_{3}}=\left(-\alpha_{0}+\alpha_{1}\right)\left(u_{1} w_{1}+u_{2} w_{2}\right), \\
v_{1 \tau_{3}}=\left(\alpha_{0}-\alpha_{-1}\right)\left(v_{1} w_{1}-v_{2} w_{2}\right), \\
w_{2 \tau_{3}}=\left(-\alpha_{1}+\alpha_{-1}\right)\left(u_{1} v_{2}-u_{2} v_{1}\right), \\
u_{2 \tau_{3}}=\left(\alpha_{1}-\alpha_{0}\right)\left(u_{2} w_{1}-u_{1} w_{2}\right), \\
v_{2 \tau_{3}}=\left(\alpha_{0}-\alpha_{-1}\right)\left(v_{2} w_{1}+v_{1} w_{2}\right) .
\end{array}\right.\right.\right.
$$

This system has also two polynomial first integrals of second order

$$
I_{1}=w_{1} w_{2}+u_{1} v_{2}-v_{1} u_{2}, \quad I_{2}=w_{1}^{2}+2 u_{1} v_{1}-w_{2}^{2}+2 u_{2} v_{2} .
$$

The traces of the powers of $q$ are expressed in terms of these two integrals. It is easy to check that all the symmetries commute and $I_{1}, I_{2}$ are first integrals for all symmetries. Thus, Lie algorithm is also applicable for this system.

The next example differs from the previous one since algebra $\mathcal{G}$ is not $\mathbb{Z}$-graded.

Example 3. Let $\mathcal{G}$ be the same algebra as in Example 2. Consider

$$
\begin{gathered}
\mathcal{G}_{0}=\left\{\left(\begin{array}{cccc}
0 & w_{1} & 0 & 0 \\
-w_{1} & 0 & 0 & 0 \\
0 & 0 & k w_{1} & 0 \\
0 & 0 & 0 & -k w_{1}
\end{array}\right)\right\}, \\
M=\left\{\left(\begin{array}{cccc}
0 & l w_{2} & 0 & v_{1} \\
-l w_{2} & 0 & 0 & v_{2} \\
-v_{1} & -v_{2} & w_{2} & 0 \\
0 & 0 & 0 & -w_{2}
\end{array}\right)\right\},
\end{gathered}
$$




$$
N=\left\{\left(\begin{array}{cccc}
0 & 0 & u_{1} & 0 \\
0 & 0 & u_{2} & 0 \\
0 & 0 & 0 & 0 \\
-u_{1} & -u_{2} & 0 & 0
\end{array}\right)\right\}
$$

where $k$ and $l$ are parameters such that $k l \neq 1$. It is clear that the hypothesis of Theorem is satisfied. Then equation (15) can be rewritten as

$$
\left\{\begin{array}{l}
w_{1 t}=\frac{\alpha_{1}-\alpha_{-1}}{1-k l}\left(-l u_{1} v_{1}+u_{2} v_{1}-u_{1} v_{2}-l u_{2} v_{2}\right) \\
u_{1 t}=\left(\alpha_{1}-\alpha_{0}\right)\left(k u_{1}-u_{2}\right) w_{1}+\left(\alpha_{1}-\alpha_{-1}\right)\left(u_{1}-l u_{2}\right) w_{2}, \\
v_{1 t}=\left(\alpha_{0}-\alpha_{-1}\right)\left(k v_{1}+v_{2}\right) w_{1} \\
w_{2 t}=\frac{\alpha_{1}-\alpha_{-1}}{1-k l}\left(u_{1} v_{1}-k u_{2} v_{1}+k u_{1} v_{2}+u_{2} v_{2}\right), \\
u_{2 t}=\left(\alpha_{1}-\alpha_{0}\right)\left(u_{1}+k u_{2}\right) w_{1}+\left(\alpha_{1}-\alpha_{-1}\right)\left(l u_{1}+u_{2}\right) w_{2}, \\
v_{2 t}=\left(\alpha_{-1}-\alpha_{0}\right)\left(v_{1}-k v_{2}\right) w_{1} .
\end{array}\right.
$$

This system possesses the same linear infinitesimal symmetries as the system in Example 2. It also has the quadratic symmetry

$$
\left\{\begin{aligned}
w_{1 \tau} & =-\frac{\left(l^{2}+1\right)\left(u_{1} v_{1}-k u_{2} v_{1}+k u_{1} v_{2}+u_{2} v_{2}\right)}{k l-1}, \\
u_{1 \tau} & =\left(k^{2}+1\right)\left(l u_{2} w_{1}-u_{1} w_{1}\right)- \\
& -\frac{k\left(\alpha_{0}+\alpha_{1}-2 \alpha_{-1}\right)+2 l\left(\alpha_{1}-\alpha_{-1}\right)+k l^{2}\left(\alpha_{0}-\alpha_{1}\right)}{\alpha_{1}-\alpha_{-1}} u_{1} w_{2}+ \\
& +\frac{\alpha_{0}-\alpha_{1}+2 k l\left(\alpha_{1}-\alpha_{-1}\right)+l^{2}\left(\alpha_{0}+\alpha_{1}-2 \alpha_{-1}\right)}{\alpha_{1}-\alpha_{-1}} u_{2} w_{2} \\
& =-\frac{\left(\alpha_{-1}-\alpha_{0}\right)\left(l^{2}+1\right)\left(k v_{1}+v_{2}\right) w_{2}}{\alpha_{1}-\alpha_{-1}}, \\
v_{1 \tau} & =\frac{k^{2}+2 k l-1}{k l-1}\left(u_{1} v_{2}-u_{2} v_{1}\right)-\frac{k^{2} l-2 k-l}{k l-1}\left(u_{1} v_{1}+u_{2} v_{2}\right) \\
w_{2 \tau} & =-\left(k^{2}+1\right)\left(l u_{1} w_{1}+u_{2} w_{1}\right)- \\
u_{2 \tau} & -\frac{\alpha_{0}-\alpha_{1}+2 k l\left(\alpha_{1}-\alpha_{-1}\right)+l^{2}\left(\alpha_{0}+\alpha_{1}-2 \alpha_{-1}\right)}{\alpha_{1}-\alpha_{-1}} u_{1} w_{2}- \\
& -\frac{k\left(\alpha_{0}+\alpha_{1}-2 \alpha_{-1}\right)+2 l\left(\alpha_{1}-\alpha_{-1}\right)+k l^{2}\left(\alpha_{0}-\alpha_{1}\right)}{\alpha_{1}-\alpha_{-1}} u_{2} w_{2} \\
v_{1}-\alpha_{-1} & =\frac{\left(\alpha_{-1}-\alpha_{0}\right)\left(l^{2}+1\right)\left(v_{1}-k v_{2}\right) w_{2}}{\alpha_{2}} .
\end{aligned}\right.
$$

Moreover, the system also has the following quadratic first integrals

$$
\begin{gathered}
I_{1}=-2 u_{1} v_{1}-2 u_{2} v_{2}+\left(k w_{1}+w_{2}\right)^{2}-\left(w_{1}+l w_{2}\right)^{2}, \\
I_{2}=u_{2} v_{1}-u_{1} v_{2}-\left(k w_{1}+w_{2}\right)\left(w_{1}+l w_{2}\right) .
\end{gathered}
$$

We observe that $I_{1}$ is the trace of $q^{2}$. It is easy to make sure that Lie algorithm is applicable in the present case as well. 
Remark 3. We observe that some first integrals for systems in Examples 1-3 are not uniquely defined in the complex plane. For instance,

$$
H_{2}=u^{\alpha_{0}-\alpha_{-1}} v^{\alpha_{0}-\alpha_{1}}
$$

is the first integral for (18). The system in Example 2 has the first integral of the form $I=f\left(u_{1}, u_{2}, v_{1}, v_{2}\right)$. It is easy to make sure that such integral should satisfy two equations $X(f)=0$ and $Y(f)=0$, where $X, Y$ are introduced by the formulae

$$
\begin{aligned}
& X=\left(\alpha_{1}-\alpha_{0}\right)\left(u_{1} \frac{\partial}{\partial u_{2}}-u_{2} \frac{\partial}{\partial u_{1}}\right)+\left(\alpha_{-1}-\alpha_{0}\right)\left(v_{1} \frac{\partial}{\partial v_{2}}-v_{2} \frac{\partial}{\partial v_{1}}\right), \\
& Y=\left(\alpha_{1}-\alpha_{0}\right)\left(u_{1} \frac{\partial}{\partial u_{1}}+u_{2} \frac{\partial}{\partial u_{2}}\right)-\left(\alpha_{-1}-\alpha_{0}\right)\left(v_{1} \frac{\partial}{\partial v_{1}}+v_{2} \frac{\partial}{\partial v_{2}}\right) .
\end{aligned}
$$

Solving this system of two partial differential equations, we find two simplest solutions

$$
\begin{gathered}
I_{3}=\tan \left(\frac{\alpha_{-1}-\alpha_{0}}{\alpha_{1}-\alpha_{0}} \arctan \frac{u_{1}}{u_{2}}-\arctan \frac{v_{1}}{v_{2}}\right), \\
I_{4}=\left(u_{1}^{2}+u_{2}^{2}\right)^{\frac{\alpha_{-1}-\alpha_{0}}{\alpha_{1}-\alpha_{0}}}\left(v_{1}^{2}+v_{2}^{2}\right) \sin ^{2}\left(\frac{\alpha_{-1}-\alpha_{0}}{\alpha_{1}-\alpha_{0}} \arctan \frac{u_{2}}{u_{1}}-\arctan \frac{v_{2}}{v_{1}}\right) .
\end{gathered}
$$

We observe that the number of polynomial symmetries and first integrals in Examples 1-3 equals to the number of independent variables and it gives the possibility to integrate these systems by quadratures by means of Lie algorithm. We do not know whether it is true for all the systems described in Theorem in Section 2.

\section{ACKNOWLEDGMENTS}

The authors express their gratitude to I.Z. Golubchik, V.V. Sokolov for the attention to the work and to A.I. Zobnin for the attention to the work and help in the work on the text. O.S. was partially supported by RFBR grant 11-01-00341-a.

\section{BIBLIOGRAPHY}

1. B. Kostant. Quantization and representation theory. Proc. SRC/LMS Res. Symp., Oxford 1977, Lond. Math. Soc. Lect. Note Ser. 34, 287-316 (1979).

2. M.A. Semenov-Tyan-Shanskii. What is a classical r-matrix? Funkts. anal. pril. 17:4, 17-33 (1983). [Funct. Anal. Appl. 17:4, 259-272 (1983).]

3. P.I. Golod. Hamiltonian systems on orbits of affine Lie groups and nonlinear integrable equations. in "Physics of multi-particle systems", Naukova Dumka, Kiev, 7, 30-39 (1985). (in Russian).

4. I.Z. Golubchik, V.V. Sokolov. On some generalizations of the factorization method. Teor. matem. fiz. 110:3, 339-350 (1997). [Theor. Math. Phys. 110:3, 267-276 (1997).]

5. A.S. Miščenko, A.T. Fomenko. Euler equations on finite-dimensional Lie groups. Izv. AN SSSR. Ser. matem. 42:2, 396-415 (1978). [Math USSR Izv. 12:2, 371-389 (1978).]

6. A.G. Reiman, M.A. Semenov-Tyan-Shanskii. Integrable systems. Theoretical group approach. RKhD, Izhevsk (2003). (in Russian).

7. A.V. Borisov, I.S. Mamaev. Dynamics of rigid body. NITS, Regulyarnaya i Khaoticheskaya Dinamika, Moscow (2001). (in Russian).

8. A.P. Veselov. On integrability conditions for the Euler equations on so(4). Dokl. AN SSSR. 270:6, 1298-1300 (1983). [Sov. Math. Dokl. 27, 740-742 (1983).]

9. V.V. Sokolov. One class of quadratic so(4)-Hamiltonians // Dokl. RAN, 394:5, 602-605 (2004). [Dokl. Math. 69:1, 108-111 (2004).]

10. V.V. Sokolov, T. Wolf. New integrable quadratic Hamiltonians on so(4) and so(3,1). J. Phys. A: Math. Gen. 39:8, 1915-1936 (2006). 
Rushaniya Akhyarovna Atnagulova,

Bashkir State Pedagogical University named after M.Akmulla,

October rev. st., 3a,

450000, Ufa, Russia

E-mail: rushania2009@yandex.ru

Ol'ga Vladimirovna Sokolova,

Faculty of Mechanics and Mathematics, Lomonosov Moscow State University,

GSP-1, Vorob'evy gory, MSU,

119991, Moscow, Russia

E-mail: Olga.Efimovskaya@gmail.com 